\title{
Review and performance of the Dose Profiler, a particle therapy treatments online monitor.
}

\author{
G. Traini ${ }^{\mathrm{b}, \mathrm{c}, \mathrm{e}}$, I. Mattei ${ }^{\mathrm{a}}$, G. Battistoni ${ }^{\mathrm{a}}$, M. G. Bisogni ${ }^{\mathrm{g}, \mathrm{j}, *}$, M. De Simoni ${ }^{\mathrm{b}, \mathrm{c}}$, Y. Dong ${ }^{\mathrm{a}, \mathrm{h}}$ \\ A. Embriaco ${ }^{a}$, M. Fischetti ${ }^{f, c}$, M. Magi ${ }^{f, c}$, C. Mancini-Terracciano ${ }^{\text {b,c }}$, M. Marafini ${ }^{\mathrm{e}, \mathrm{c}}$, \\ R. Mirabelli ${ }^{\mathrm{b}, \mathrm{c}}$, S. Murarog ${ }^{\mathrm{g}}$, V. Patera ${ }^{\mathrm{f}, \mathrm{c}, \mathrm{e}}$, A. Schiavi ${ }^{\mathrm{f}, \mathrm{c}}$, A. Sciubba ${ }^{\mathrm{f}, \mathrm{c}, \mathrm{e}}$, E. Solfaroli \\ Camillocci $^{\mathrm{b}, \mathrm{c}, \mathrm{i}}$, S. M. Valle ${ }^{\mathrm{a}}$, A. Sarti ${ }^{\mathrm{f}, \mathrm{d}, \mathrm{e}}$ \\ ${ }^{a}$ INFN Sezione di Milano, Italy \\ ${ }^{b}$ Dipartimento di Fisica, Sapienza Università di Roma, Italy \\ ${ }^{c}$ INFN Sezione di Roma, Italy \\ ${ }^{d}$ INFN Sezione dei Laboratori di Frascati, Roma, Italy \\ ${ }^{e}$ Museo Storico della Fisica e Centro Studi e Ricerche "E. Fermi", Roma, Italy \\ ${ }^{f}$ Dipartimento di Scienze di Base e Applicate per l'Ingegneria, Sapienza Università di Roma, Italy \\ ${ }^{g}$ INFN Sezione di Pisa, Italy \\ ${ }^{h}$ Dipartimento di Fisica, Università degli Studi di Milano, Italy \\ ${ }^{i}$ Scuola di Specializzazione in Fisica Medica, Sapienza Università di Roma, Roma, Italy \\ ${ }^{j}$ Dipartimento di Fisica "E. Fermi", Università di Pisa, Pisa, Italy
}

\begin{abstract}
Particle therapy (PT) can exploit heavy ions (such as He, $\mathrm{C}$ or $\mathrm{O}$ ) to enhance the treatment efficacy, profiting from the increased Relative Biological Effectiveness and Oxygen Enhancement Ratio of these projectiles with respect to proton beams. To maximise the gain in tumor control probability a precise online monitoring of the dose release is needed, avoiding unnecessary large safety margins surroundings the tumor volume accounting for possible patient mispositioning or morphological changes with respect to the initial CT scan. The Dose Profiler (DP) detector, presented in this manuscript, is a scintillating fibres tracker of charged secondary particles (mainly protons) that will be operating during the treatment, allowing for an online range monitoring. Such monitoring technique is particularly promising in the context of heavy ions PT, in which the precision achievable by other techniques based on secondary photons detection is limited by the environmental background during the beam delivery. Developed and built at the SBAI department of "La Sapienza", within the INSIDE collaboration and as part of a Centro Fermi flagship project, the DP is a tracker detector specifically designed and planned for clinical applications inside a PT treatment room. The DP operation in clinical like conditions has been tested with the proton and carbon ions beams of Trento proton-therapy center and of the CNAO facility. In this contribution the detector performances are presented, in the context of the carbon ions monitoring clinical trial that is about to start at the CNAO centre.
\end{abstract}

Keywords: Instrumentation for heavy-ion therapy, Particle tracking detectors, Radiation monitoring 


\section{Introduction}

Particle Therapy (PT) is a tumor control technique that makes use of charged ion beams targeting the tumor region inside a patient to maximize the biological damage to the cancer cells while sparing the surrounding healthy tissues [1]. These characteristics are directly related to the energy loss mechanism of charged ions inside matter, which results in the Bragg Peak (BP) shape of the associated dose release. The spatial selectivity as well as the biological damage inflicted to the cells are furthermore enhanced when ions with $\mathrm{Z}>1$ are used, due to their higher Relative Biological Effectiveness (RBE) and the reduced Oxygen Enhancement Ratio (OER) [2], [3]. However, in PT several sources of beam range uncertainty may affect the therapy outcome, e.g. CT scan mis-calibrations, uncertainties in the HU to stopping power conversion, or inter-fraction patient mis-positioning and morphological changes occurred in the patient with respect to the initial treatment planning [4]. Therefore, large safety margins $(2.5-3 \%$ of the range $+1-3 \mathrm{~mm})$ [5, 6] are implemented in the planning preserving the overall treatment efficacy. An online monitoring technique, currently missing in the clinical practice, would allow for a significantly margin reduction, minimizing the Normal Tissues Complication Probability (NTCP).

In the context of proton therapy there are techniques being developed targeting the detection of secondary prompt gamma radiation [7], [8], or annihilation photons emitted by the beam-induced $\beta^{+}$activation of the patient nuclei [9, 10, 11]. When applied to the monitoring of heavier ions $\mathrm{PT}$, like $\mathrm{He}, \mathrm{C}$ and $\mathrm{O}$ the precision achievable on the $\mathrm{BP}$ position is significantly reduced, because of the large background represented by secondary neutrons and beam-uncorrelated photons. However, for the $\mathrm{Z}>1$ ions treatments the detection of charged secondary particles seems particularly promising, as the charged fragments production remains significant even at large angles with respect to the beam incoming direction [12]. This is a direct consequence of the fragments production mechanism: the high energy fragments that are able to escape the patient body are mainly the products of the projectile fragmentation. The fragment yields for different beams impinging on thick targets have been studied and reported elsewhere [13, 14, 15, 16]. Furthermore, the detection of charged fragments, when compared to the photon detection, can be carried out in a nearly background free environment and can be performed with high efficiency.

The Dose Profiler (DP) is a multi-layer scintillating fibre tracker developed as part the INSIDE (INnovative Solution for In-beam DosimEtry) dual-mode integrated monitoring system [17, which provides an online PET device and the DP charged tracker to monitor the treatments at the Centro Nazionale per l'Adroterapia Oncologica (CNAO, Pavia, Italy). The DP has been funded also as a flagship CentroFermi project dedicated to the online monitoring of charged PT treatments with $\mathrm{Z}>1$ ions. The preliminary DP design and its expected performance has already been documented in [18]. In this manuscript the final optimized design of the detector, whose construction ended in May 2017 at the "Scienze di

\footnotetext{
*Corresponding author at: Dipartimento di Fisica, Università degli studi di Pisa, Largo Bruno Pontecorvo, 356127 PISA

Email address: Giuseppina.Bisogni@pi.infn.it (M. G. Bisogni)
} 
Base e Applicate per l'Ingegneria" Department of the "La Sapienza" University, is presented. A detector characterization campaign has been carried out in the Trento APSS (Azienda Provinciale per i Servizi Sanitari) centre and in the CNAO centre using both protons and carbon ions beams. Particular care has been made in order to test the DP operation and performance in clinical-like conditions using beams of clinical energy and intensity and an anthropomorphic phantom as target.

The details on the DP design and the experimental setup of the operation test are given in $\S 2$, the details of the event reconstruction and particle tracking software can be found in $\S 4$ and the obtained results, in terms of detector operation and performance, are summarized in $\S 5$.

\section{The Dose Profiler}

The choice of the DP detection technology has been driven by the constraints posed by the operation inside a treatment room in clinical like conditions. The Dose Profiler detector has been designed and optimized to fulfill the following requirements:

- High detection efficiency. The charged secondary fragments produced in PT treatments are preferentially emitted at forward angles, as they are the product of the projectile fragmentation. However, light fragments such as protons and deuterons, can be emitted at large angles with respect to the beam axis [15]. The kinetic energy spectrum of particles escaped from the patient, heavily depends on their production point and on the path towards the detector (density and thickness of traversed matter). A highly efficient detection has to be ensured for protons and deuterons with kinetic energy in the range of $50 \div 200 \mathrm{MeV}$.

- Large acceptance. The achievable range monitoring precision crucially depends on the amount of detected particles during a PT treatment. A careful optimization of the detector has been performed to maximize the statistics that can be collected by the device, while keeping its size and the distance from the patient compliant with the clinical operation in a treatment room.

- High rate capability. The therapeutical Carbon ion beam intensity at CNAO is of the order of $10^{8} \mathrm{~s}^{-1}$, resulting in an incoming rate of charged fragments crossing the detector area as large as few hundreds of $\mathrm{kHz}$ (depending on detector position and primary beam energy). The related tracks need to be reconstructed and the full event analysis and the spatial emission spectrum construction needs to be performed in less then $10 \mu \mathrm{s}$, posing strong constraints on the affordable Dead Time (DT).

- The backtracking resolution needs to be lower than the average multiple scattering undergone by the fragments inside the patient $(\sim 5 \div 10 \mathrm{~mm})$.

According to the aforementioned considerations, plastic scintillating fibres that are readout by SiPMs (Silicon Photomultipliers) have been chosen as detecting active media. Such 
technology is fully compatible with a safe operation in a treatment room (no gas or high voltage are needed), providing the required spatial resolution and a high detection efficiency for proton and heavier charged fragment of the energies of interest for PT monitoring applications. Moreover, the produced fast signal does not affect significantly the read-out dead time.

The detector details are discussed in $\S 2.1$, where the geometry layout (number of fibre layers, their positioning, the global detector positioning with respect to the patient) and readout optimization are reported. In this paragraph, the details on the detector construction and initial tests are given. The experimental setup used for the detector characterization using particle beams is presented in $\S 3.1$ and $\S 3.2$. Protons and carbon ions of different energies have been used in the Trento APSS and CNAO facilities to benchmark the detector response to secondary fragments. Finally, the Monte Carlo (MC) simulation used to optimize the detector setup will be outlined in $\S 2.4$, underlining the work done to take properly into account the measured detector efficiency and cross talk between readout channels when computing the detector tracking resolution for data - MC comparison purposes.

\subsection{Detector design}

The DP layout and detection principle are shown in figure1. The spatial reconstruction of the charged fragments trajectory is performed using double-cladding, squared plastic scintillator fibres ${ }^{1}, 500 \mu \mathrm{m}$ in side, arranged in 8 subsequent planes fixed by means of an aluminum frame. A charged particle traversing the fibres releases part of its kinetic energy producing a light signal (the light yield of the fibres used for the DP construction is $\sim 8000$ photons $/ \mathrm{MeV}$ ). A small fraction of the scintillation light is then guided by total internal reflection (trapping efficiency of $7.2 \%$ [19]) to the fibre ends, where it is detected by SiPMs. The crossed fibres signal is used to identify the coordinates of the incoming particle interaction points, revealing the particle trajectory. Each plane is composed by two adjacent fibre layers arranged in orthogonal directions, providing the $\mathrm{x}-\mathrm{y}$ coordinates, whereas the $\mathrm{z}$ coordinate along the detector axis (precisely known and constrained by the detector mechanical support) is taken as the position of the fibre planes centres. The fibre layers contain 384 fibres $19.2 \mathrm{~cm}$ long, resulting in a plane active area of $19.2 \times 19.2 \mathrm{~cm}^{2}$.

The chosen inter-plane distance is $2 \mathrm{~cm}$, as a compromise among the maximum angular acceptance, the mechanical constraints posed by the integration with the clinical room environment and the minimum backtracking resolution. The whole detector volume, including the read-out system, is $\sim 30 \times 30 \times 30 \mathrm{~cm}^{3}$. The detector will be operated, within the INSIDE project, in a single cart that will hold two PET heads and the DP for the bimodal simultaneous monitoring of PT beams. The integration with the PET detector sets a limit on how close to the patient the detector can be placed. In the operating position, the DP will be placed $\sim 50 \mathrm{~cm}$ away from the isocentre, with a tilt angle of $60^{\circ}$ with respect to the beam direction, resulting in a solid angle covered by the detector of $\sim 0.15 \mathrm{sr}$. However, different distances from the isocentre have been explored using a dedicated test beam setup,

\footnotetext{
${ }^{1}$ Model BFC-12, provided by Saint-Gobain
} 



Figure 1: Left: schematic view of the DP, illustrating the fragments detection principle. The yellow squares identify the energy released in the fibres layers (in azure), whereas the particle trajectory is shown in green. Right: view of the Dose Profiler layers during the assembly phase showing the aluminum frames and the fibre layers.

to evaluate the tracking resolution and particle rate dependence on the DP distance from the isocentre (see $\S 5.2$.

\subsection{Read-out system}

The fibres are read-out by means of $1 \mathrm{~mm}^{2}$ active area Hamamatsu S12571-050P SiPMs, that are coupled to $\sim 2$ fibres as the SiPM-fibre alignment is not ideal, because of some unavoidable small dead-space between the fibres derived by the assembling procedure. The SiPMs are arranged on rectangular boards housing two parallel lines of 96 SiPMs each. Four boards are needed to read-out two subsequent planes in both the $\mathrm{x}$ and $\mathrm{y}$ view, forming a $b i$ plane unit, as shown in figure 2. The SiPMs arrangement, with adjacent fibre pairs coupled to SiPMs in two opposed boards, was defined to compensate for the dead space in between adjacent SiPMs $(1 \mathrm{~mm})$ needed to house the sensor packaging. The SiPMs control and read-out is managed by 32 channel, custom integrated circuits named BASIC32ADC [20], specifically developed for the SiPM read-out in medical applications. The ASIC internal 8 bit ADC provides the integrated charge, which is registered for each channel that exceeds an adjustable threshold. A threshold fine tuning has been performed in laboratory tests with cosmic radiation (mostly muons) and light injection (performed using LEDs), finding the configuration which maximises the trigger efficiency against charged particles using MIPs, while keeping a negligible noise rate.

An operation bias voltage of SiPM around $66 \mathrm{~V}$ has been set to have a uniform SiPM gain of $\sim 1.2 \times 10^{5}$. Ninety-six BASIC32ADC ASICs, organized in sixteen boards, are needed to read-out all the 3072 detector channels. Each board is interfaced with a control FPGA used for the ASICs read-out and configuration. In order to maximise the signal to noise SiPM ratio, all the boards are cooled with aluminum plates connected to a circuit with 
circulating water kept at $\sim 12{ }^{\circ} \mathrm{C}$ by a coolen ${ }^{2}$. The total detector power to be dissipated is around 100 watts. A board named concentrator, hosting a commercial embedded system ${ }^{3}$, is used as a master unit to generate and to distribute the trigger signal, to collect the data from the read-out boards and to manage the communication via ethernet link to an external workstation used for the data storage and processing. The concentrator also interacts with the Dose Delivery System (DDS) of the CNAO [21, recording the treatment voxel and slice index during the beam delivery for the event processing (more details are provided in $\S 4.2$ ).
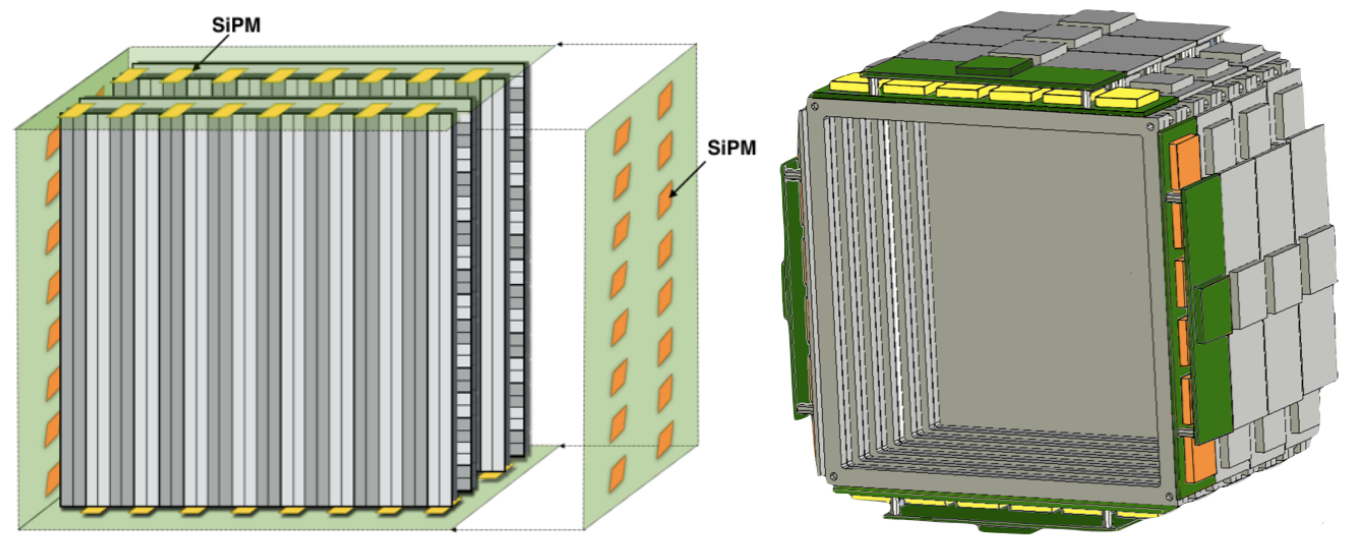

Figure 2: Left: Schematic view of the fibres readout performed using SiPMs. As the minimum distance between two adjacent SiPMs on the readout board is $1 \mathrm{~mm}$, to minimise the impact of such dead-space the readout of adjacent pairs of fibres is performed by SiPMs placed on the two opposite sides of each plane. The SiPMs are shifted, on the two sides, exactly by $1 \mathrm{~mm}$ allowing the read-out of all the fibres. The top and bottom side SiPMs used for the read-out of the fibres are highlighted respectively in yellow and red. Right: schematic view of the readout boards. For the very first bi-plane BASICs are shown in yellow and orange, respectively for the $\mathrm{x}$ and $\mathrm{y}$ views, while the read-out FPGAs and the boards are shown in green.

\subsection{Trigger and $D A Q$}

The DP has been designed implementing two different customizable triggering strategies: a stand-alone triggering mode in which the information from each ASIC is combined and used to decide if the event is of interest, and an external triggering mode in which the boards readout is activated by an external input. The former will be used during the clinical operation. The time coincidence of over-threshold signals in at least one SiPM in both of the $\mathrm{x}-\mathrm{y}$ of a bi-planes (defined in $\S 2.2$ is used as trigger elementary unit. To select charge fragments traversing the detector, a valid signal in all the four bi-planes units is required. As soon as the trigger signal is asserted, the ASIC read-out starts, and the data are sent to the concentrator for the event-building through 16 serial lines with a transfer speed of $100 \mathrm{Mbit} / \mathrm{s}$. The data transfer strategy exploits the bunched CNAO beam time structure: when the beam is being delivered (in-spill) the data are just saved in the concentrator

\footnotetext{
${ }^{2}$ Model JULABO F250

${ }^{3}$ Model AVnet Picozed 7020
} 
memory, while being transferred to the external workstation during the inter-spill period $(\sim 2 \mathrm{~s})$, reducing the dead-time contribution due to the ethernet transmission. With such read-out architecture the total DT per event is of the order of few $\mu$ s (see $\S 5.1$ ), allowing to sustain an average $50 \mathrm{kHz}$ trigger rate, with peaks that can reach $\mathrm{O}(100) \mathrm{kHz}$.

\subsection{Simulation}

A Monte Carlo (MC) simulation was implemented using the FLUKA software [22, 23] to optimise the tracker layout and evaluate the expected performances. The interactions of protons and other charged fragments with energies in the range that is relevant for particle therapy applications were studied using the MC simulation carefully tuned to reproduce the experimental setup described in $\S 3.1$ and $\S 3.2$. Main interest of the study was the evaluation of the detector response, and in particular the detector efficiency and tracking resolution. The protons used for the detector benchmarking and development were either monochromatic (in energy) or produced with the different energies and directions as predicted by the nuclear models implemented in FLUKA and used to describe the interactions of a Carbon ion beam with a given target. In this second condition, we do expect an impact on the data-MC comparison due to the differences in the model adopted within FLUKA to generate the energy and production point of secondary charged particles with respect to the acquired data.

The obtained results, presented in $\S 5$, are in good agreement with the expectations from the MC simulations as detailed, whenever relevant, in the following paragraphs.

\section{Experimental setup}

\subsection{The Trento APSS setup}

Protons represent the largest component in the population of the fragments escaping from the patient in a Carbon ion PT treatment, with a small contamination of deuteron and tritons [15, 16]. The DP response to protons with energies from $\sim 45 \mathrm{MeV}$ to $\sim 230 \mathrm{MeV}$ has been studied using proton beams at the experimental cave of the Trento APSS facility [24. The proton beams have been shot on the detector entrance surface, placed $50 \mathrm{~cm}$ away from the room isocenter, with a parallel direction to the detector axis. The beams intensity was of the order of $\sim 10^{3} \mathrm{p} / \mathrm{s}$. To perform a full scan of the DP active volume, the detector was placed on a remotely controllable table, able to shift along the the $\mathrm{x}-\mathrm{y}$ directions with millimetre-level precision steps. A sketch and a picture of the experimental setup are shown in Figure 3 .

The DP was operated for most of the time in self-triggering mode, testing the running stability and measuring the efficiency and resolution for protons. Two thin plastic scintillator read-out by commercial Photomultipliers (STS1 and STS2) with size $5 \times 5 \times 1 \mathrm{~cm}^{3}$ were used to provide a trigger to test the external-trigger DP operational mode.

\subsection{The CNAO setup}

In order to test the DP performance in clinical like conditions, several tests have been performed at the CNAO treatment centre, which can provide Carbon ion beams with the 

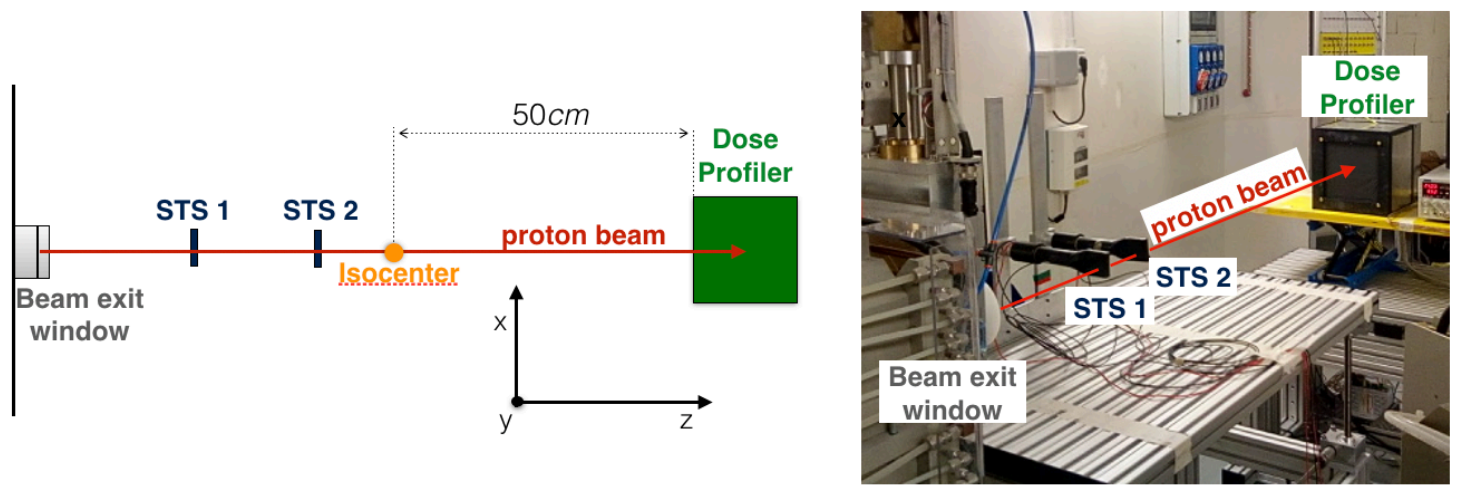

Figure 3: Schematic view (left) and picture (right) of the Trento experimental setup. The beam exit window is marked in grey. Before hitting the DP (marked in green) entrance window the beam crossed two scintillator detectors (STS1 and STS2, in blue). The detector was placed at a distance of $50 \mathrm{~cm}$ from the isocenter (marked in orange in the left schematic view). When testing the DP acquisition using the self-triggering mode, the scintillator detectors were removed.

intensity and energy routinely used for PT treatments (up to $\sim 400 \mathrm{Mev} / \mathrm{u}$ ). The experimental setup is shown in figure 4. The DP was placed on the patient couch side, forming an angle of $60^{\circ}$ with the beam direction, pointing to the room isocenter. It was held by a provisional cart with wheels, movable at different distances from the target. The device height was fixed at $120 \mathrm{~cm}$ above ground, to vertically align the room isocentre with the DP centre. Two different targets were used to produce secondary fragments:

- An anthropomorphic head phantom moulded of tissue-equivalent materials 4 used to reproduce clinical-like conditions. The phantom lied on the couch and the beam impinged the skullcap centre placed at $\sim 60 \mathrm{~cm}$ from the beam exit window. The distance of the isocentre from the DP was fixed to $50 \mathrm{~cm}$.

- A small plastic sphere (4 mm diameter), used as a point-like source of charged fragments to evaluate the back-tracking resolution. The sphere was centred in the isocentre position, while the DP was moved to study the distance dependence of the resolution in the $30 \div 80 \mathrm{~cm}$ range.

The targets were irradiated with single monochromatic Carbon ion beams of $180 \mathrm{MeV} / \mathrm{u}$, $220 \mathrm{MeV} / \mathrm{u}, 280 \mathrm{MeV} / \mathrm{u}$ and $320 \mathrm{MeV} / \mathrm{u}$ energy. The DP was operated in stand-alone triggering mode for all the described configurations.

\section{Event reconstruction}

For each collected event the DAQ system registers all the over threshold SiPMs (pixels in the following). In this paragraph, the data-processing flow needed to perform the tracking reconstruction and the fragment emission point measurement is described.

\footnotetext{
${ }^{4} \mathrm{RANDO} \cap$
} 


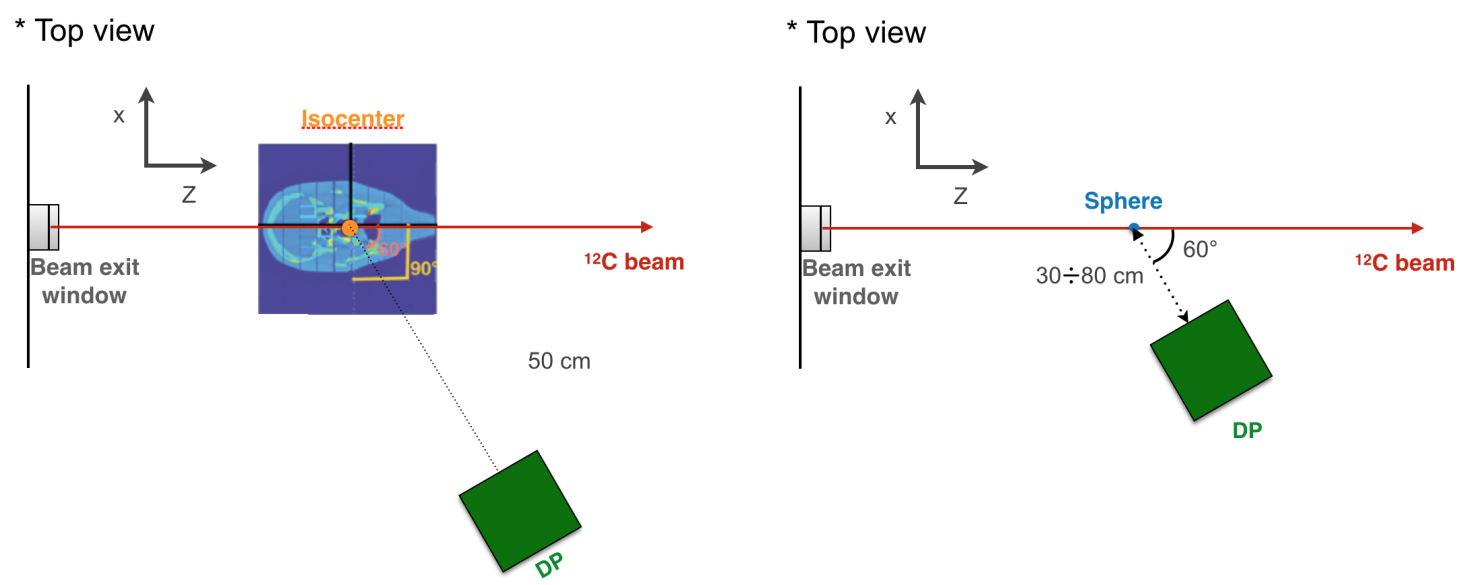

Figure 4: Schematic view of the CNAO experimental setup (not to scale) with the anthropomorphic target (left) and with a small plastic spherical target simulating a point-like source of secondary fragments for the back-pointing resolution measurement (right).

\subsection{Pixel clustering}

The DP fibres are not totally optically insulated: a double cladding technology has been used to reduce the light loss, increasing the total reflection probability. In spite of that, in case of a very large light yield output some light can also cross and enter nearby fibres (optical cross-talk). Furthermore, as the fibres are not perfectly geometrically aligned with the readout SiPMs, it is possible that a given fibre is readout by two SiPMs on different detector sides. For these reasons, the pixels with a signal over threshold are clustered, assigning to a given cluster all the pixels that are belonging to nearby positions in space. The combination of light cross talk and geometrical mismatches results into an average cluster size of $\sim 1.5$ pixels per cluster, as shown in Figure 5. The spectra of the number of pixels per cluster has been measured at the Trento APSS facility with protons of different kinetic energies $E_{k}$, superimposed in different colors in Figure 5. The expected dependence of the cluster size on the fragment energy is observed: the cluster size is larger when the energy release inside the fibres increases. The cluster position in the transverse plane, taken as the arithmetic average of the pixels position, is then used for the track reconstruction described here-after.

\subsection{Track reconstruction}

The reconstruction of the fragment track exploits the Hough transform [25] to recognize straight lines in the identified cluster spatial distribution, separately for the $\mathrm{x}-\mathrm{z}$ and $\mathrm{y}-\mathrm{z}$ views. Track candidates are required to have at least four aligned clusters in both views: such requirement has been tuned against the data and $\mathrm{MC}$ event samples to maximise the track reconstruction efficiency, while minimising the number of background tracks made of noise clusters (the number of reconstructed clusters that are not associated to any track is $\sim 2.5$ per event in each view). The track parameters are then evaluated by performing a linear chisquare fit. The Multiple Scattering interactions occurred by the fragments within 


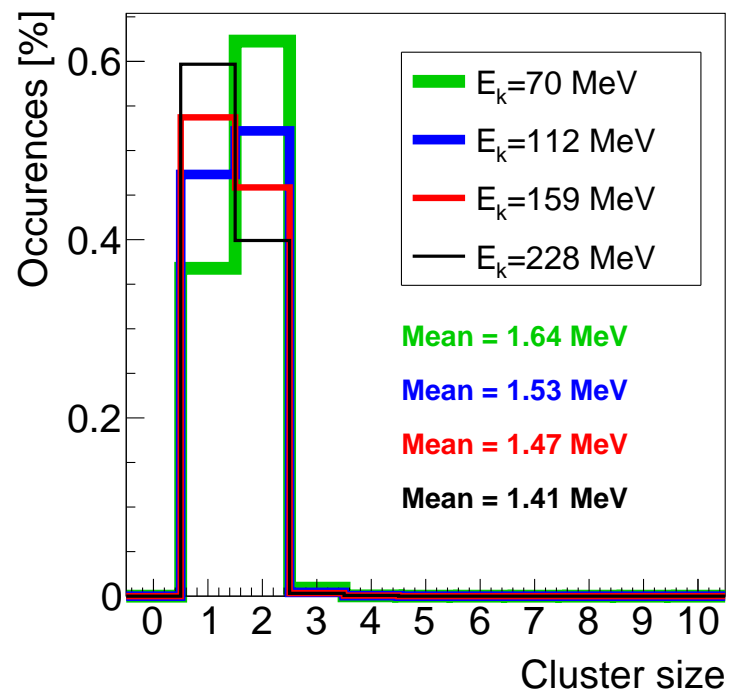

Figure 5: Cluster size measured using protons of different kinetic energies at the Trento APSS centre. The cluster size is shown only for track associated clusters and it is defined as the number of pixels which compose a cluster associated to a reconstructed track.

the fibres does not significantly affect the linear fit procedure at all the detected proton energies. The smallness of the effect is directly related to the small inter-plane distance and the high measured cluster size. An event reconstruction example is shown in Figure6, where a single fragment is crossing the full detector active volume.

The DP capability of detecting multiple fragments is shown in figure 7 , where the reconstruction of an event in which two particles have simultaneously crossed the detector is shown. However, such events are indeed rare as their occurrence is less than $10 \%$ of the total in clinical like conditions. In figure 8 the track multiplicity in an event sample collected using carbon ions beams at $280 \mathrm{MeV} / \mathrm{u}$ impinging on the anthropomorphic head-phantom at $\mathrm{CNAO}$ is shown.

\subsection{Emission point evaluation}

The fragment back-tracking is then performed using the measured track parameters and transforming them in the treatment room reference frame. The $3 \mathrm{D}$ coordinates of the fragment production point are estimated using the point of closest approach (POCA) of the track with respect to the incoming beam particles direction. In clinical operations the beam direction will be known combining the treatment plan information and the decoded data acquired from the DDS (as previously described in section $\S 2.2$ ). The latter provides, during the treatment, the information about the voxel and slice that are being targeted. The reconstructed fragments POCAs distribution will be used to monitor the beam range inside the patient as discussed in [15] and [12]. 


\section{$\mathrm{X}-\mathrm{Z}$ view}

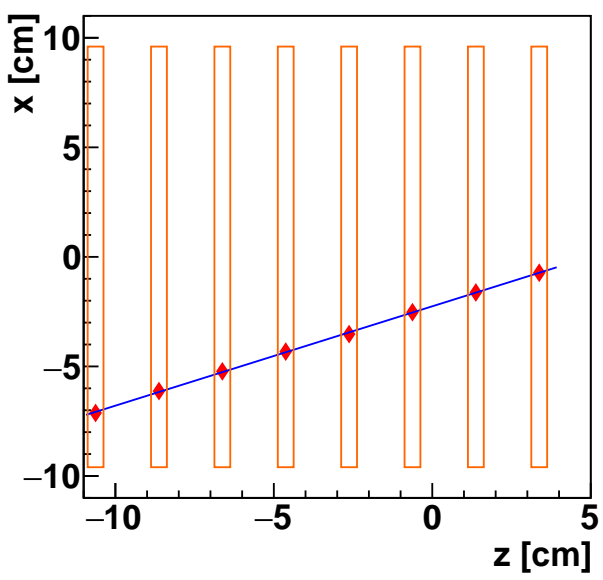

Y-Z view



Figure 6: Tracking of a charged fragment traversing the DP. The eight empty orange rectangles are representing the eight fibre planes in the $x-z$ (left) and $y-z$ (right) view. The red markers are showing the position of the reconstructed clusters, and the reconstructed trajectory is super-imposed (blue line). The small detector noise results in such clean event displays in which the contamination from spurious clusters is negligible.
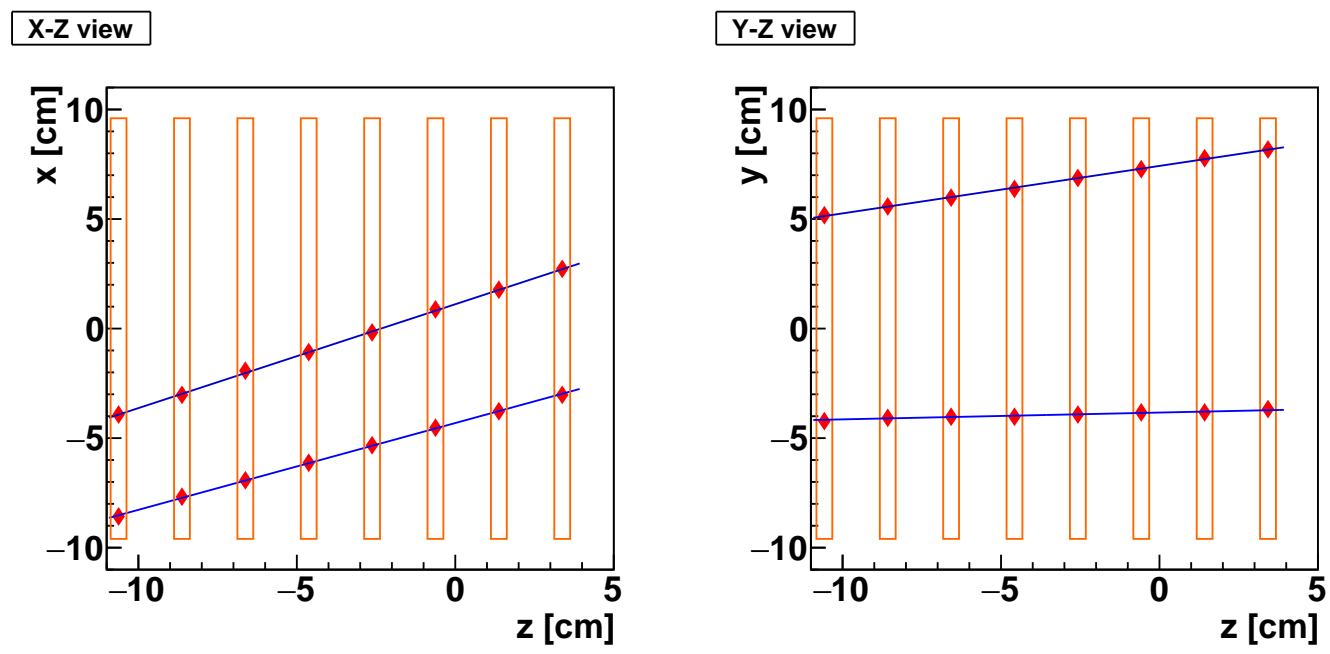

Figure 7: Display of a rare event in which two fragments are simultaneously crossing the detector active volume. The $\mathrm{x}-\mathrm{z}$ and $\mathrm{y}-\mathrm{z}$ views of the detector are shown respectively on the left and on the right. 


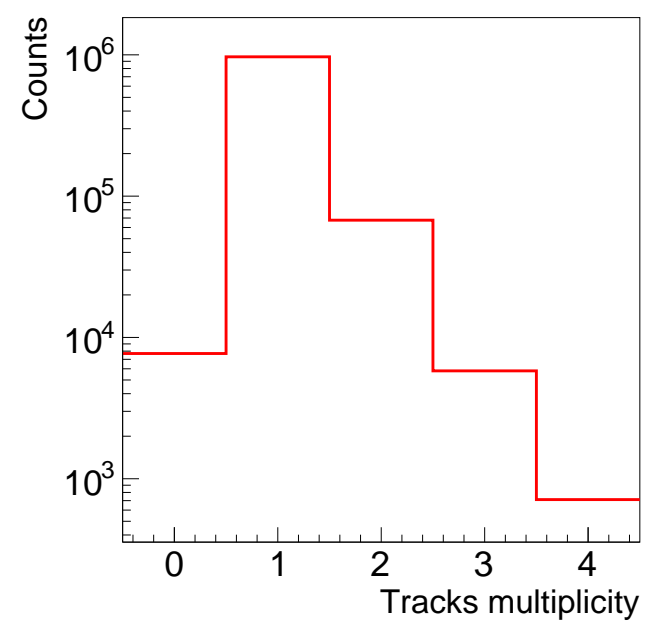

Figure 8: Track multiplicity observed in an event sample collected using Carbon ions beams used for the treatment of a patient impinging on an anthropomorphic head phantom target.

\section{Detector perfomances}

As the signal detected by the DP in clinical operations will be mostly made by protons, the benchmarking of the detector performances has been firstly performed using proton beams. The detector response has been characterized using protons of known energy, used also to tune the track reconstruction algorithms and test the detector readout capability and hardware stability. As second step, we used Carbon ion beams interacting with different targets, using the DP for secondary fragments detection. In such data-taking campaigns it was possible to evaluate the DAQ performance in clinical like conditions, the tracking efficiency and back-tracking resolution and the kinetic spectra of the detected fragments. The obtained results are presented in the following paragraphs.

\subsection{The detector operation: DAQ rate and hardware stability}

The DP must be operated in clinical conditions, where very large detected charged fragments rates are expected since $\sim 10^{8}$ ions are delivered within a single spill, which lasts of the order of few seconds. The hardware stability under these conditions has been tested delivering a Carbon ion treatment plan of a patient treated at CNAO on the head phantom target. The obtained results are shown in figure 9.

The measured DAQ rate reaches values as large as $\sim 150 \mathrm{kHz}$, depending on the beam energy and on the material traversed by the fragments before reaching the detector. The DAQ stability in time has been checked by comparing the results obtained repeating several times the treatment delivery using the same target conditions: the number of acquired triggers was stable with a negligible variation (less than 1\%). Neither event loss nor data corruption were observed. The detector dead time per triggered event is entirely dominated by the ASIC data readout chain, and it has been measured using a counter with $100 \mathrm{MHz}$ 

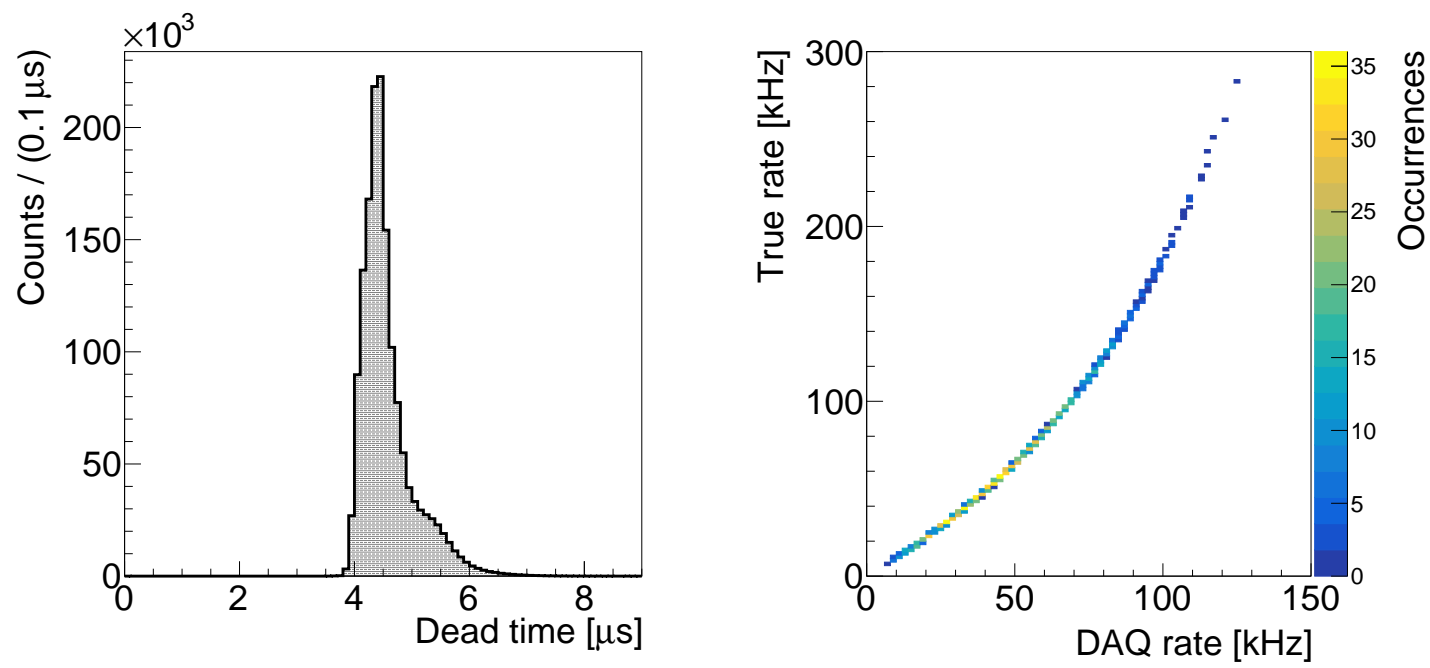

Figure 9: Left: distribution of the dead time per event, measured delivering a Carbon ion treatment plan on an anthropomorphic head phantom target. Right: true event rate, assessed using the average DT shown on the left, shown as function of the measured DAQ rate.

frequency, embedded in the concentrator board. The measured mean DT value of $\sim 4 \mu \mathrm{s}$ matches the expected one. When taking into account the DP mean dead time, the production rates can be measured: values reaching few hundreds of $\mathrm{kHz}$ were observed.

\subsection{Back-tracking resolution}

In clinical operation, the accuracy on the fragments emission position is limited by the multiple scattering interactions undergone by the fragment with the patient body along the exit path. This effect introduces an uncertainty of the order of $5 \div 10 \mathrm{~mm}$, depending on the crossed material. Then, a detector back-pointing resolution lower than $10 \mathrm{~mm}$ matches the PT monitoring requirements, having placed the target at a distance of $\sim 50 \mathrm{~cm}$.

The resolution on the fragment emission point, projected along the beam axis $(z)$, has been studied detecting the fragments produced by Carbon ions of $280 \mathrm{MeV} / \mathrm{u}$ kinetic energy impinging on a small plastic sphere (4 mm diameter) target, placed at variable distances from the DP. The detected fragments were back-tracked towards their emission point with the procedure described in $\S$ 4, reconstructing the sphere dimensions in the transverse plane, whose z-projection is shown in figure 10 (left). A function defined as the sum two gaussians and a 4th degree polynomial has been used to parametrize the signal, formed by the secondary fragments produced within the sphere, and the background fragments produced in air, respectively. The standard deviation of the double gaussian is taken as the spatial resolution.

The resolution along the beam direction is shown in Figure 10 (right) as a function of the distance from the target in the range between 30 and $80 \mathrm{~cm}$. At the operational distance from the iso-centre $(\sim 50 \mathrm{~cm})$ the measured tracking resolution is $\sim 7 \mathrm{~mm}$, matching the requirement set for PT applications, with a value that is consistent with the expectations 

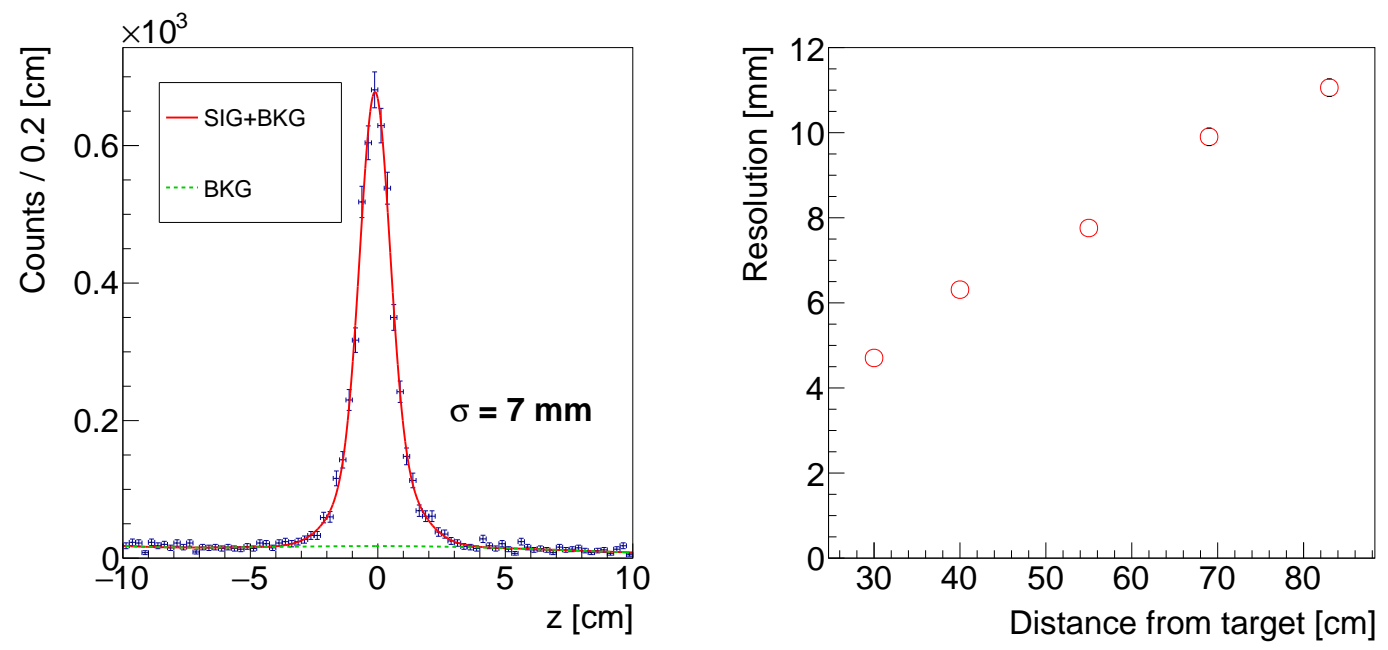

Figure 10: Left: Distribution of the reconstructed coordinate along the beam axis, measured using the fragments produced by a Carbon ion beam at $280 \mathrm{MeV} / \mathrm{u}$ impinging on a plastic sphere target . The DP was placed at a distance from the target of $\sim 50 \mathrm{~cm}$. Right: DP tracking resolution as a function of the distance from the target.

obtained using dedicated FLUKA MC simulations.

\subsection{Charged particles detection efficiency}

The DP mean detection efficiency for each fibre plane $\varepsilon_{i}$ has been measured delivering a Carbon ions treatment plan on the anthropomorphic phantom target. To estimate the efficiency of the the i-th layer $\left(\varepsilon_{i}\right)$, the collected events in the data sample has been tracked without using the information from the plane under test, for each plane. Such tracks were hence extrapolated in the skipped plane, searching for the presence of a fired cluster within a fiducial distance of $1.5 \mathrm{~mm}$, corresponding to $3 \sigma$ of the residuals distribution. The efficiency has been evaluated as the ratio of the number of found cluster in the layer under test $\left(N_{\mathrm{Cl}}^{i}\right)$ and the total number of reconstructed tracks traversing it $\left(N_{\operatorname{Tr}}^{i}\right)$, as described in the following equation.

$$
\varepsilon_{i}=\frac{N_{\mathrm{Cl}}^{i}}{N_{\mathrm{Tr}}^{i}}
$$

This method provides an unbiased method to compute the efficiency as the information from the plane of interest is not used for the track reconstruction. Despite the aforementioned efficiency definition involves tracking reconstruction, this does not affect the measurement thanks to the high tracking reconstruction algorithm robustness, resulting in a tracking efficiency of the order of 1.The results obtained when testing the overall layer efficiency, are shown in Figure 11 for all the eight layers of each view.

The measured detection efficiency, $\sim 90 \%$ for all the layers, is consistent with the expectations resulting from the fibre double cladding ( $\sim 5 \%$ of the fibre size), from the small 

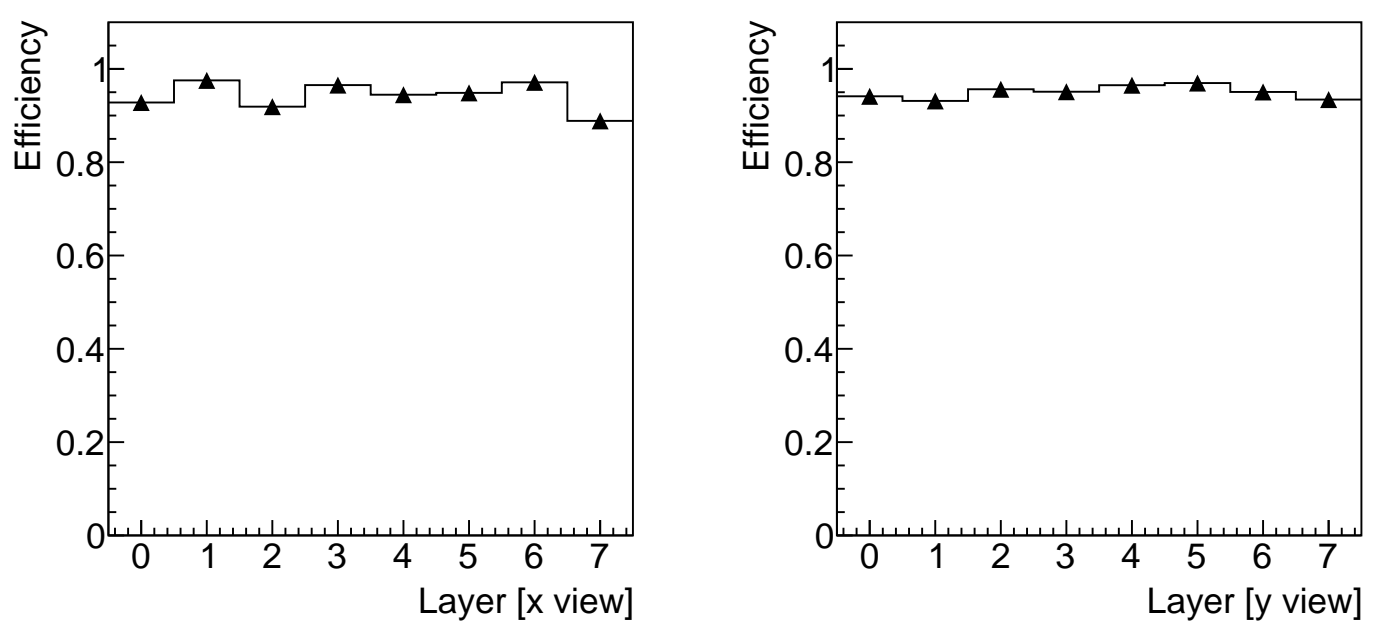

Figure 11: DP average detection efficiency as a function of the fibre layer. The results are presented separately for the different detection views: $\mathrm{x}-\mathrm{z}$ view results are shown on the left, while $\mathrm{y}-\mathrm{z}$ results on the right. The statistical uncertainty, shown in the plot, is too small to be appreciated.

SiPM-fibre unavoidable misalignment, and from the presence of non active materials between the adjacent fibres used in the assembling phase, hence reducing the overall detector active volume (see 2.2). However, the trigger efficiency is not affected by such tiny drop, as the trigger relies on the logic described in $\S 2.3$, resulting in an overall trigger efficiency of $\sim 98 \%$. A systematic uncertainty has been assigned to each measured efficiency value by comparing the results obtained repeating several times the treatment delivery. However, the computed uncertainties are within the marker size of the figure 11 . The observed fluctuations could be reasonably explained with the different SiPM/fibre alignment for each plane.

\subsection{Secondary charged fragments kinetic energy spectra}

By using the monochromatic proton beams of the Trento APSS centre (see $\S$ 3.1) it was also possible to calibrate the detector response to protons of different energy, using the average charge produced in a detector layer $Q_{\text {rel }}$, defined as

$$
Q_{\mathrm{rel}}=\frac{\sum_{i=1}^{N} Q_{\mathrm{SiPM}}^{i}}{N_{\text {lay }}}
$$

where $Q_{\mathrm{SiPM}}^{i}$ is the measured charged by the i-th pixel belonging to a reconstructed track, and $N_{\text {lay }}$ is the number of crossed fibre layers. The $Q_{\text {rel }}$ distributions are shown for different proton kinetic energies $\left(\mathrm{E}_{\text {kin }}\right)$ in Figure 12 (left), pointing out a measured resolution in the $15-20 \%$ range.

A $Q_{\text {rel }}-E_{\text {kin }}$ calibration has been performed with the parametrization shown in figure 12 (right). Such calibration has been used to measure the kinetic energy $\left(\mathrm{E}_{k}\right)$ of the fragments produced by carbon ion beams of different energies impinging on a anthropomorphic head 
phantom, with the assumption of a negligible presence of $\mathrm{Z}>1$ fragments. The obtained results are shown in Figure 13 .
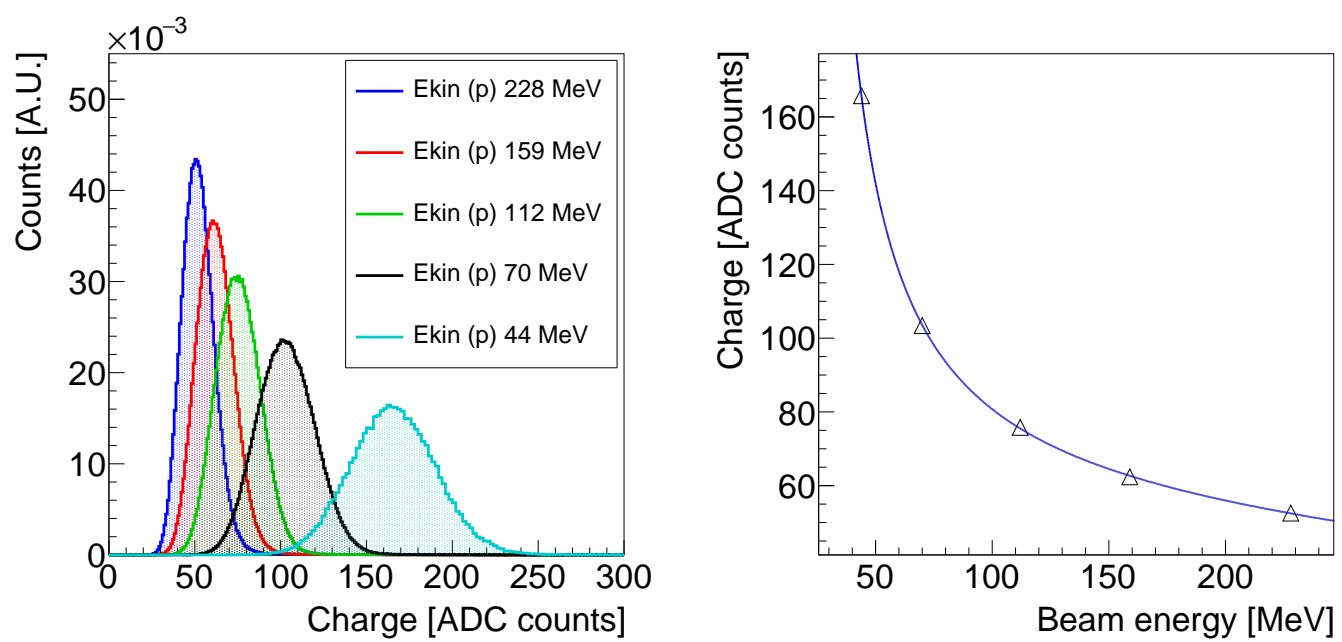

Figure 12: Left: The distributions of $Q_{\text {rel }}$ for proton beams of different energies. Right: charge-kinetic energy calibration obtained with a data-driven based model.

The observed spectra are consistent with the expectations: no fragments are detected below $\sim 30 \mathrm{MeV}$, as a minimum energy is required to cross at least 6 fibre planes to produce a trigger signal $\S 2.3$, and the mean energy increases (slightly) with the increasing incoming beam energy. Each spectrum is obtained using the same number of impinging Carbon ion $\left(10^{9}\right)$, the different spectra amplitudes reflect the different fragment production at different energies due to the different range.

\section{Conclusions}

The Dose Profiler detector is a charged fragments tracker developed for beam range online monitoring in PT treatments. The operational tests performed since its completion in may 2017 demonstrated that the design performances have been achieved. The DP is a stable, fully functional and efficient detector capable of performing the fragment tracking at the design rates (up to $100 \mathrm{kHz}$ ). The measured back-tracking resolution per fragment matches the design value ( $7 \mathrm{~mm}$ at $50 \mathrm{~cm}$ from the patient) and is hence suitable for charged fragments monitoring applications. The data collected with anthropomorphic phantom in clinical like conditions proved that the DP is ready to be safely and efficiently operated during the clinical trial phase that will start in 2019. The data collected during the clinical trial will be used to assess the final precision achievable in range monitoring applications and in spotting morphological changes and/or misplacement of the patient with respect to the planned treatment. 


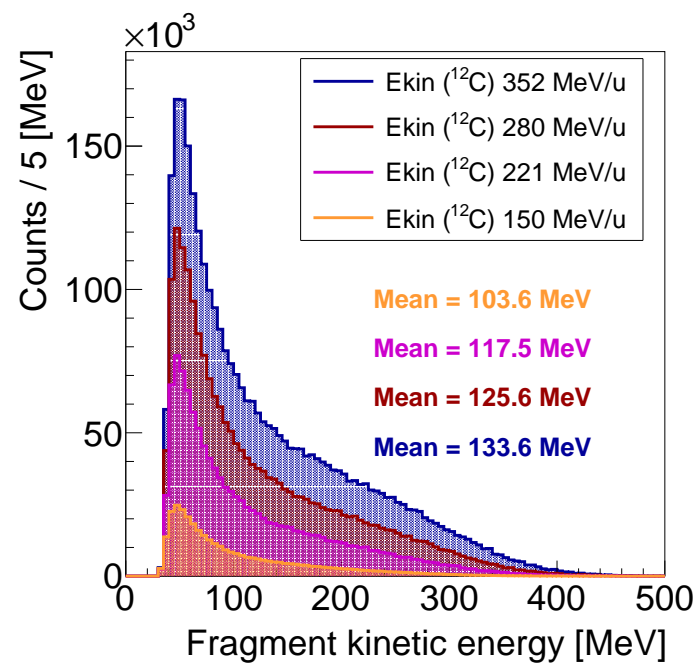

Figure 13: Kinetic energy distributions for fragments exiting from an antropomorphic phantom on which carbon ions of different energies are impinging. The results are shown for incoming ions of 151, 221, 280 and $352 \mathrm{MeV} / \mathrm{u}$ kinetic energy. The average energy of the fragments increases slightly, with the increasing beam energy, as expected.

\section{Acknowledgments}

We would like to acknowledge all the personnel of the CNAO and APSS centres that provided us support during the operational tests performed using carbon and proton beams. In particular we would like to thank M. Ciocca, M. Pullia, G. Venchi, E. Fiorina of the CNAO centre and F. Tommasino, C. La Tessa, M. Rovituso from the University of Trento, and M. Durante from GSI.

\section{References}

[1] M. Durante, R. Orecchia, J. Loeffler, Charged-particle therapy in cancer: Clinical uses and future perspectives, Nature Reviews Clinical Oncology 14 (8) (2017) 483-495. doi:10.1038/nrclinonc. 2017.30.

[2] H. Paganetti, Relative biological effectiveness (RBE) values for proton beam therapy. variations as a function of biological endpoint, dose, and linear energy transfer, Physics in Medicine and Biology 59 (22) (2014) R419-R472. doi:10.1088/0031-9155/59/22/r419.

[3] F. Tommasino, E. Scifoni, M. Durante, New ions for therapy, International Journal of Particle Therapy 2 (3) (2015) 428-438. doi:10.14338/IJPT-15-00027.1.

[4] H. Paganetti, Range uncertainties in proton therapy and the role of monte carlo simulations, Physics in Medicine and Biology 57 (11) (2012) R99-R117. doi:10.1088/0031-9155/57/11/r99.

[5] N. Okonogi, M. Wakatsuki, S. Kato, K. Karasawa, H. Kiyohara, S. Shiba, D. Kobayashi, T. Nakano, T. Kamada, M. Shozu, the Working Group of Gynecological Tumors, Clinical outcomes of carbon ion radiotherapy with concurrent chemotherapy for locally advanced uterine cervical adenocarcinoma in a phase 1/2 clinical trial (protocol 1001), Cancer Medicine 7 (2) (2018) 351-359. doi:10.1002/cam4. 1305 . 
[6] D. Bridges, H. Kawamura, T. Kanai, Probabilistic dose distribution from interfractional motion in carbon ion radiation therapy for prostate cancer shows rectum sparing with moderate target coverage degradation, PLOS ONE 13 (8) (2018) 1-19. doi:10.1371/journal.pone.0203289.

[7] Y. Xie, E. H. Bentefour, G. Janssens, J. Smeets, F. V. Stappen, L. Hotoiu, L. Yin, D. Dolney, S. Avery, F. O'Grady, D. Prieels, J. McDonough, T. D. Solberg, R. A. Lustig, A. Lin, B.-K. K. Teo, Prompt gamma imaging for invivo range verification of pencil beam scanning proton therapy, International Journal of Radiation Oncology*Biology*Physics 99 (1) (2017) 210 - 218. doi:https://doi.org/10. 1016/j.ijrobp.2017.04.027.

[8] F. Hueso-González, M. Rabe, T. A. Ruggieri, T. Bortfeld, J. M. Verburg, A full-scale clinical prototype for proton range verification using prompt gamma-ray spectroscopy, Physics in Medicine \& Biology 63 (18) (2018) 185019. doi:10.1088/1361-6560/aad513

[9] K. Parodi, On- and off-line monitoring of ion beam treatment, Nuclear Instruments and Methods in Physics Research Section A: Accelerators, Spectrometers, Detectors and Associated Equipment 809 (2016) 113 - 119, advances in detectors and applications for medicine. doi:https://doi.org/10. 1016/j.nima.2015.06.056.

[10] V. Ferrero, E. Fiorina, M. Morrocchi, F. Pennazio, G. Baroni, G. Battistoni, N. Belcari, N. Camarlinghi, M. Ciocca, A. Del Guerra, M. Donetti, S. Giordanengo, G. Giraudo, V. Patera, C. Peroni, A. Rivetti, M. D. da Rocha Rolo, S. Rossi, V. Rosso, G. Sportelli, S. Tampellini, F. Valvo, R. Wheadon, P. Cerello, M. G. Bisogni, Online proton therapy monitoring: clinical test of a silicon-photodetector-based in-beam pet, Scientific Reports 8 (1) (2018) 4100. doi:10.1038/s41598-018-22325-6.

[11] E. Fiorina, V. Ferrero, F. Pennazio, G. Baroni, G. Battistoni, N. Belcari, P. Cerello, N. Camarlinghi, M. Ciocca, A. D. Guerra, M. Donetti, A. Ferrari, S. Giordanengo, G. Giraudo, A. Mairani, M. Morrocchi, C. Peroni, A. Rivetti, M. D. R. Rolo, S. Rossi, V. Rosso, P. Sala, G. Sportelli, S. Tampellini, F. Valvo, R. Wheadon, M. Bisogni, Monte carlo simulation tool for online treatment monitoring in hadrontherapy with in-beam pet: A patient study, Physica Medica 51 (2018) 71 - 80. doi:https://doi.org/10.1016/j.ejmp.2018.05.002.

[12] S. Muraro, G. Battistoni, F. Collamati, E. De Lucia, R. Faccini, F. Ferroni, S. Fiore, P. Frallicciardi, M. Marafini, I. Mattei, S. Morganti, R. Paramatti, L. Piersanti, D. Pinci, A. Rucinski, A. Russomando, A. Sarti, A. Sciubba, E. Solfaroli-Camillocci, M. Toppi, G. Traini, C. Voena, V. Patera, Monitoring of hadrontherapy treatments by means of charged particle detection, Frontiers in Oncology 6 (2016) 177. doi:10.3389/fonc.2016.00177.

[13] E. Haettner, H. Iwase, M. Krmer, G. Kraft, D. Schardt, Experimental study of nuclear fragmentation of 200 and $400 \mathrm{MeV} / \mathrm{u} 12 \mathrm{c}$ ions in water for applications in particle therapy, Physics in Medicine and Biology 58 (23) (2013) 8265-8279. doi:10.1088/0031-9155/58/23/8265.

[14] K. Gunzert-Marx, H. Iwase, D. Schardt, R. S. Simon, Secondary beam fragments produced by $200 \mathrm{MeV}$ $\mathrm{u}-112 \mathrm{c}$ ions in water and their dose contributions in carbon ion radiotherapy, New Journal of Physics 10 (7) (2008) 075003. doi:10.1088/1367-2630/10/7/075003.

[15] L. Piersanti, F. Bellini, F. Bini, F. Collamati, E. D. Lucia, M. Durante, R. Faccini, F. Ferroni, S. Fiore, E. Iarocci, C. L. Tessa, M. Marafini, I. Mattei, V. Patera, P. G. Ortega, A. Sarti, C. Schuy, A. Sciubba, M. Vanstalle, C. Voena, Measurement of charged particle yields from PMMA irradiated by a 220 $\mathrm{MeV} / \mathrm{u} 12 \mathrm{cbeam}$, Physics in Medicine and Biology 59 (7) (2014) 1857-1872. doi:10.1088/0031-9155/ $59 / 7 / 1857$.

[16] A. Rucinski, G. Battistoni, F. Collamati, E. D. Lucia, R. Faccini, P. M. Frallicciardi, C. ManciniTerracciano, M. Marafini, I. Mattei, S. Muraro, R. Paramatti, L. Piersanti, D. Pinci, A. Russomando, A. Sarti, A. Sciubba, E. S. Camillocci, M. Toppi, G. Traini, C. Voena, V. Patera, Secondary radiation measurements for particle therapy applications: charged particles produced by 4 he and $12 \mathrm{c}$ ion beams in a PMMA target at large angle, Physics in Medicine \& Biology 63 (5) (2018) 055018. doi:10.1088/ $1361-6560 /$ aaa36a

[17] B. Maria Giuseppina, A. Andrea, B. Giuseppe, B. Nicola, . C. Niccoló, C. Piergiorgio, C. Silvia, D. G. Alberto, F. Alfredo, F. Veronica, F. Elisa, G. Giuseppe, K. Eleftheria, M. Matteo, P. Francesco, P. Cristiana, P. Maria Antonietta, P. Giovanni, R. Angelo, R. Manuel D., R. Valeria, S. Paola, S. Giancarlo, 
W. Richard, Inside in-beam positron emission tomography system for particle range monitoring in hadrontherapy, Journal of Medical Imaging 4 (1) (2016) 1 - 12 -12. doi:10.1117/1. JMI.4.1.011005.

[18] G. Traini, G. Battistoni, A. Bollella, F. Collamati, E. D. Lucia, R. Faccini, F. Ferroni, P. M. Frallicciardi, C. Mancini-Terracciano, M. Marafini, I. Mattei, F. Miraglia, S. Muraro, R. Paramatti, L. Piersanti, D. Pinci, A. Rucinski, A. Russomando, A. Sarti, A. Sciubba, M. Senzacqua, E. Solfaroli-Camillocci, M. Toppi, C. Voena, V. Patera, Design of a new tracking device for on-line beam range monitor in carbon therapy, Physica Medica 34 (2017) 18 - 27. doi:https://doi.org/10.1016/j.ejmp.2017.01.004.

[19] Saint-Gobain, Scintillating products, scintillating optical fibers, Brochure (2010).

URL ttps://www.crystals.saint-gobain.com/sites/imdf.crystals.com/files/documents/ fiber-brochure.pdf

[20] F. Ciciriello, F. Corsi, F. Licciulli, C. Marzocca, G. Matarrese, E. Chesi, E. Nappi, A. Rudge, J. Seguinot, A. Del Guerra, Basic32_adc, a front-end asic for sipm detectors, in: 2013 IEEE Nuclear Science Symposium and Medical Imaging Conference (2013 NSS/MIC), 2013, pp. 1-6. doi: 10.1109/NSSMIC.2013.6829781

[21] S. Giordanengo, M. A. Garella, F. Marchetto, F. Bourhaleb, M. Ciocca, A. Mirandola, V. Monaco, M. A. Hosseini, C. Peroni, R. Sacchi, R. Cirio, M. Donetti, The cnao dose delivery system for modulated scanning ion beam radiotherapy, Medical Physics 42 (1) (2015) 263-275. doi:10.1118/1.4903276.

[22] A. Ferrari, P. R. Sala, A. Fasso, J. Ranft, FLUKA: A multi-particle transport code (Program version 2005), CERN-2005-010, SLAC-R-773, INFN-TC-05-11.

[23] G. Battistoni, J. Bauer, T. T. Boehlen, F. Cerutti, M. P. W. Chin, R. Dos Santos Augusto, A. Ferrari, P. G. Ortega, W. Kozowska, G. Magro, A. Mairani, K. Parodi, P. R. Sala, P. Schoofs, T. Tessonnier, V. Vlachoudis, The fluka code: An accurate simulation tool for particle therapy, Frontiers in Oncology 6 (2016) 116. doi:10.3389/fonc.2016.00116.

[24] F. Tommasino, M. Rovituso, S. Fabiano, S. Piffer, C. Manea, S. Lorentini, S. Lanzone, Z. Wang, M. Pasini, W. Burger, C. L. Tessa, E. Scifoni, M. Schwarz, M. Durante, Proton beam characterization in the experimental room of the trento proton therapy facility, Nuclear Instruments and Methods in Physics Research Section A: Accelerators, Spectrometers, Detectors and Associated Equipment 869 (2017) 15 - 20. doi:https://doi.org/10.1016/j.nima.2017.06.017.

[25] R. O. Duda, P. E. Hart, Use of the hough transformation to detect lines and curves in pictures, Commun. ACM 15 (1) (1972) 11-15. doi:10.1145/361237.361242. 